\section{THU0013 IL-17A AND IL-17F ARE SECRETED BY ENTHESIS T CELLS AND SYNERGIZE WITH TNF TO INDUCE CCL20 FROM ENTHESEAL STROMAL CELLS}

Charlie Bridgewood $^{1}$, Tobias Russell ${ }^{1}$, Abdulla Watad ${ }^{2}$, Hannah Rowe ${ }^{1}$, Qiao Zhou', Almas Khan ${ }^{3}$, Peter Loughenbury ${ }^{3}$, Abhay S Rao ${ }^{3}$, Peter Millner ${ }^{3}$, Robert Dunsmuir ${ }^{3}$, Richard Cuthbert ${ }^{1}$, Dennis Mcgonagle ${ }^{1} .{ }^{1}$ Leeds Institute of Rheumatic and Musculoskeletal Medicine, University of Leeds, Leeds, Leeds, United Kingdom; ' ${ }^{2}$ Department of Medicine ' $B$ ', Zabludowicz Center for Autoimmune Diseases, Sheba Medical Center, Tel-Hashomer, Israel, Sackler Faculty of Medicine, Tel-Aviv University, Tel-Aviv, Tel-Aviv, Israel; ${ }^{3}$ The Leeds Teaching Hospitals NHS Trust, Leeds, United Kingdom, Leeds, United Kingdom

Background: Enthesitis or inflammation of tendon/ligament anchorage points is the cardinal lesion in spondyloarthritis $(S p A)[1]$. Human $\operatorname{SpA}$ is thought to be driven by $\mathrm{LL}-17$ producing $\mathrm{T}$-cells and can be successfully therapeutically targeted with anti-IL-17A and more recently anti-IL-17F[2]. The CCR6-CCL20 axis is thought to be crucial to tissue recruitment of IL-17 producing T-cells into tissues, however it is presently unknown if CCR6+ T-cells reside at the healthy human enthesis[3]. Moreover, the ability of IL-17 to drive CCL20 production and hence to promote further T-cell migration to the enthesis has not been tested.

Objectives: To determine if enthesis $T$ cells secrete IL-17A and IL-17F. To determine if CCR6+ T-cells reside at the normal human enthesis. To study the effect of both IL-17A and IL-17F on CCL20 induction from entheseal stromal cells.

Methods: Normal spinous process enthesis was obtained from patients undergoing spinal decompression or surgery for scoliosis correction. Following enzymatic digestion, $\mathrm{CD} 4+$ and $\mathrm{CD} 8+\mathrm{T}$ cells were stimulated with anti CD3/CD28 and IL-17A and IL-17F measured by intracellular FACS. Stromal cells were isolated from both the peri-entheseal bone (PEB) and enthesis soft tissue (ST). Stromal cells were stimulated with combinations of IL-17A or IL-17F and TNF and subsequently CCL20 was measured by ELISA. Normal PEB and ST were also analysed for the presence CCR6 + T-cells by multiparameter FACS including CD4+ and CD8

Results: Entheseal CD4+ cells secreted IL-17A and IL-17F following stimulation. When used as single agents IL-17A, IL-17F and TNF were able to induce minimal CCL20 from entheseal stromal cells, but great synergy was reported between IL-17A/TNF and IL-17F/TNF. No synergy was reported for IL-17A/IL-17F. CCR6+ CD4+ and CD8+ cells were also confirmed in the enthesis.

Figure 1: IL-17A and IL-17F synergize with TNF to induce CCL20 from Entheseal stromal cells
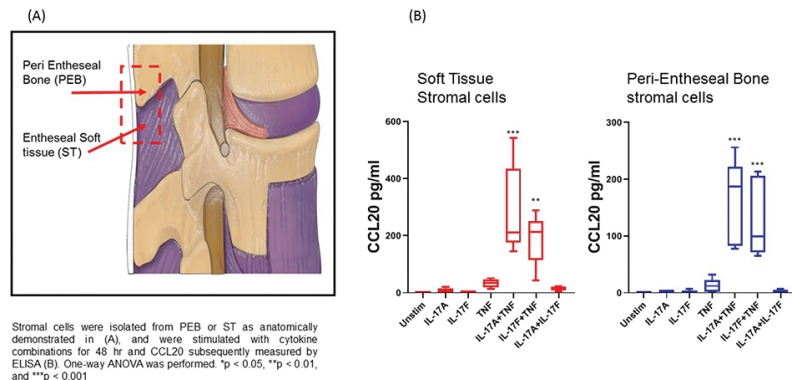

Abstract THU0013 Figure 1.

Conclusion: Resident CD4+ T cells at the enthesis secrete IL-17A and IL-17F following stimulation. The normal human enthesis contains CCR6+ $\mathrm{T}$ cell populations, suggesting a CCR6/CCL2O axis is important to entheseal homeostasis. IL-17A/F dramatically synergize with TNF to induce CCL20 from enthesis stromal cells, which would be well placed to mediate further migration of IL-17 producing lymphocytes to entheseal tissues.

\section{REFERENCES:}

[1] Bridgewood, C., et al., Spondyloarthritis: new insights into clinical aspects, translational immunology and therapeutics. Current opinion in rheumatology, 2018. 30(5): p. 526-532.

[2] Hall, A.O.H., J.E. Towne, and S.E. Plevy, Get the IL-17F outta here! Nature immunology, 2018: p. 1.
[3] Singh, S.P., et al., Human T cells that are able to produce $I L-17$ express the chemokine receptor CCR6. The Journal of Immunology, 2008. 180(1): p. 214-221.

Disclosure of Interests: Charlie Bridgewood: None declared, Tobias Rus sell Grant/research support from: PhD Project is funded by Novartis., Abdulla Watad: None declared, Hannah Rowe: None declared, Qiao Zhou: None declared, Almas Khan: None declared, Peter Loughenbury: None declared, Abhay S Rao: None declared, Peter Millner: None declared, Robert Dunsmuir: None declared, Richard Cuthbert: None declared, Dennis McGonagle Consultant for: Lilly, Novartis UCB, Speakers bureau: Lilly, Novartis UCB DOI: 10.1136/annrheumdis-2019-eular.3527

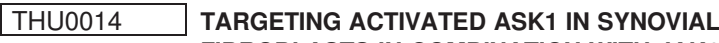 FIBROBLASTS IN COMBINATION WITH JAK1 INHIBITION ENHANCES EFFICACY IN RAT CIA}

Paqui Gonzalez-Traves, Christian Franci, Julie A. DI Paolo. Gilead Sciences, Inc., Foster City, United States of America

Background: Despite improved therapy, rheumatoid arthritis (RA) remains an area of unmet medical need. Current therapies have improved disease control by targeting inflammatory pathways. However, treatments rarely induce remission, highlighting the need for new therapies. Filgotinib (FIL) is an oral selective JAK1 inhibitor that has demonstrated clinical efficacy in RA trials. ${ }^{1-3}$ Apoptosis signal-regulating kinase 1 (ASK1) is a member of the MAP3K family that activates $\mathrm{p} 38$ and $\mathrm{c}$-jun and has recently been shown to modulate human RA fibroblast-like synoviocyte (FLS) invasion, proliferation, and migration in vitro. ${ }^{4}$ We hypothesize that by dual targeting of JAK-dependent inflammatory pathways with FIL, and ASK1 signaling in FLS with an ASK1 inhibitor, we can demonstrate an increase in efficacy in a rat collagen-induced arthritis (CIA) model.

Objectives: To evaluate the individual and combination activity of JAK1 and ASK1 inhibition in the rat CIA model by oral dosing with FIL and an ASK1 inhibitor

Methods: The in vivo efficacy of FIL and an ASK1 inhibitor were tested individually or in combination in a therapeutic rat CIA model. Dosing was initiated at the onset of disease (day 11) and continued until day 18 Efficacy evaluations were based on animal body weights, daily ankle caliper measurements, ankle diameter (expressed as area under the curve), terminal hind paw weights, and histopathology of the ankles and knees.

Results: Administration of FIL individually significantly reduced ankle diameter and final paw weights by $51 \%$ and $52 \%$, respectively $(p<0.05)$ There was no change in body weight loss or in histological measurements with treatment. Conversely, ASK1 inhibition did not reduce inflammation as measured by ankle diameter or paw swelling, but resulted in a $48 \%$ reduction in ankle histopathological score $(p<0.05)$. The combination of FIL and the ASK1 inhibitor showed significantly greater effects on all measured parameters including paw weight ( $81 \%$ reduction), ankle diameter $(78 \%$ reduction), and ankle and knee histopathology scores (69\% and $87 \%$ reduction, respectively) than either agent alone. Body weight loss was also significantly reduced with the combination, and increased toward normal weight gain compared to the monotherapy arms. Conclusion: Combining FIL with ASK1 inhibition significantly improved clinical and histopathology scores, and reduced body weight loss in this model. These data suggest that simultaneously targeting JAK and ASK1 pathways can provide orthogonal activities that can enhance overall disease control.

\section{REFERENCES:}

[1] Kavanaugh A, et al. Ann Rheum Dis. 2017;76:1009-1019.

[2] Westhovens R, et al. Ann Rheum Dis. 2017;76:998-1008.

[3] MC Genovese, et al. ACR 2018, Abstract L06.

[4] Nygaard G, et al. Biochem Pharm. 2018;151:282-290.

Disclosure of Interests: Paqui Gonzalez-Traves Grant/research support from: Gilead Sciences, Inc., Employee of: Gilead Sciences, Inc., Christian Franci Shareholder of: Gilead Sciences, Inc., Employee of: Gilead Sciences, Inc., Julie A. Di Paolo Shareholder of: Gilead Sciences, Inc., Employee of: Gilead Sciences, Inc.

DOI: 10.1136/annrheumdis-2019-eular.2145 